Ciência Florestal, Santa Maria, v. 25, n. 2, p. 491-499, abr.-jun., 2015

ISSN 0103-9954

\title{
REGISTRO DE NOVAS ESPÉCIES DE FORMIGAS (HYMENOPTERA: FORMICIDAE) NO ESTADO DO RIO GRANDE DO SUL
}

\author{
REGISTER OF NEW SPECIES OF ANTS (HYMENOPTERA: FORMICIDAE) IN RIO GRANDE \\ DO SUL STATE
}

\author{
Marciane Danniela Fleck ${ }^{1}$ Edison Bisognin Cantarelli ${ }^{2}$ Fabiane Granzotto $^{3}$
}

\begin{abstract}
RESUMO
O presente trabalho objetivou registrar a ocorrência de novas espécies de formigas para o estado do Rio Grande do Sul e, com isso, contribuir para o conhecimento sobre a mirmecofauna. As coletas foram realizadas em quatro ambientes (floresta nativa, plantio de eucalipto, plantio agrícola e pastagem exótica). Para a coleta das formigas, foi utilizado o método do extrator de mini-Winkler, cujo processo consistiu em retirar $1 \mathrm{~m}^{2}$ de serapilheira, peneirá-la e acondicioná-la em extratores de mini-Winkler para a obtenção da fauna. Foram coletadas 108 espécies, representantes de 31 gêneros, 18 tribos e oito subfamílias. Destas, a floresta nativa, apresentou 90 espécies coletadas, seguida pelo plantio de eucalipto, com 65 espécies, pelo plantio agrícola e pela pastagem exótica, com 20 espécies cada. Neste trabalho, é feito o primeiro registro de ocorrência de Dorymyrmex thoracicus (Dolichoderinae), Camponotus arboreus, Camponotus melanoticus (Formicinae), Apterostigma madidiense, Apterostigma sp. complexo pilosum, Cyphomyrmex major, Cyphomyrmex vorticis, Wasmannia rochai, Cephalotes pusillus, Strumigenys (Pyramica) rugithorax, Strumigenys cosmotela, Strumigenys silvestrii, Pheidole aper, Pheidole heyeri, Pheidole rugatula, Pheidole siggilata, Pheidole senilis, Pheidole sospes, Megalomyrmex pusillus (Myrmicinae) e Pachycondyla ferruginea (Ponerinae) para o Rio Grande do Sul.
\end{abstract}

Palavras-chave: extrator mini-Winkler; mirmecologia; serapilheira.

\section{ABSTRACT}

This study aimed to report the registration of new ant species in Rio Grande do Sul state and thus to contribute to the knowledge about the ant fauna (Myrmecofauna). Collections were made in four different environments, namely, native forest, eucalyptus plantations, agricultural plantation and exotic pasture. We used the mini-Winkler method of extraction to collect the ants. We removed 1 square meter $\left(1 \mathrm{~m}^{2}\right)$ of litter, sifted it and packed it in mini-Winkler extractors to obtain the fauna. We collected 108 species, which represent 31 genera, 18 tribes and 8 subfamilies. The native forest presented 90 collected species, the eucalyptus plantation presented 65 species, and the agricultural plantation and the exotic pasture presented 20 species each. We have registered for the first time the following species in the state of Rio Grande do Sul: Dorymyrmex thoracicus (Dolichoderinae), Camponotus arboreus, Camponotus melanoticus (Formicinae), Apterostigma madidiense, Apterostigma sp. pilosum complex, Cyphomyrmex major, Cyphomyrmex vorticis, Wasmannia rochai, Cephalotes pusillus, Strumigenys (Pyramica) rugithorax, Strumigenys cosmotela, Strumigenys silvestrii, Pheidole aper, Pheidole heyeri, Pheidole rugatula, Pheidole siggilata, Pheidole senilis, Pheidole sospes, Megalomyrmex pusillus (Myrmicinae), and Pachycondyla ferruginea (Ponerinae). Keywords: Litter, mini-Winkler extractor, myrmecology.

1 Engenheira Florestal, Doutoranda do Programa de Pós-graduação em Engenharia Florestal, Centro de Ciências Rurais, Universidade Federal de Santa Maria, Av. Roraima, 1000, CEP 97105-000, Santa Maria (RS), Brasil. marcianedanniela@gmail.com

2 Engenheiro Florestal, Dr., Professor Associado do Departamento de Engenharia Florestal, Centro de Educação Superior Norte do Rio Grande do Sul, Universidade Federal de Santa Maria, BR386, Km 40, Linha 7 de Setembro, CEP 98400-000, Frederico Westphalen (RS), Brasil. engedison@yahoo.com

3 Engenheira Florestal, Mestranda em Engenharia de Processos, Centro de Tecnologia, Universidade Federal de Santa Maria, Av. Roraima, 1000, CEP 97105-000, Santa Maria (RS), Brasil. fabianegranzotto@yahoo.com.br

Recebido para publicação em 6/06/2012 e aceito em 30/07/2013

Ci. Fl., v. 25, n. 2, abr.-jun., 2015 


\section{INTRODUÇÃO}

As formigas constituem o maior grupo de insetos sociais, amplamente distribuídos geograficamente, sendo encontradas em áreas que vão desde regiões subpolares até o Equador, bem como em todas as ilhas oceânicas, exceto nos polos e nos mares, sendo mais abundantes em locais de clima tropical (WILSON, 1987).

Devido a sua ampla distribuição, as formigas são um dos grupos de insetos mais conhecidos e estudados (HÖLLDOBLER e WILSON, 1990), sendo, por isso, consideradas um dos principais componentes biológicos de biomassa ou abundância relativa de ambientes estruturalmente complexos, como as florestas (FITTKAU e KLINGE, 1973).

Muitas espécies são consideradas detritívoras e/ou predadoras de outros insetos, contribuindo, dessa forma, para a reciclagem dos nutrientes e influenciando a dinâmica populacional de insetos herbívoros (HÖLLDOBLER e WILSON, 1990).

Formigas são frequentemente utilizadas em estudos sobre diversidade e comunidades, por terem a vantagem de ser organismos dominantes nos ecossistemas, devido a sua importância tanto em riqueza de espécies quanto em número de indivíduos, além da relativa facilidade de coleta e da baixa mobilidade das populações (FOWLER et al., 1991).

Dadas às dimensões do Brasil, à complexidade e ao grande número de formações vegetais e seus ecótonos, o número de levantamentos faunísticos é claramente insuficiente para permitir generalizações ou mesmo listagens confiáveis de espécies, seja por ecossistema ou por localidade (BRANDÃO, 1999).

Visando contribuir ao conhecimento da mirmecofauna que ocorre no estado do Rio Grande do Sul, o presente trabalho tem por objetivo registrar a ocorrência de novas espécies de formigas.

\section{MATERIAL E MÉTODO}

O trabalho foi desenvolvido em quatro áreas no município de Frederico Westphalen, região Noroeste do estado do Rio Grande do Sul (27 $21^{\prime}$ 'S e $\left.53^{\circ} 23^{\prime} \mathrm{W}\right)$. O clima, segundo a classificação de Köppen, é do tipo subtropical (Cfa), com chuva bem distribuída durante o ano e temperatura média do mês mais quente superior a $22^{\circ} \mathrm{C}$. A precipitação média anual varia entre 1400 e $1760 \mathrm{~mm}$, bem distribuída ao longo do ano (MORENO, 1961).

O relevo do município é ocupado pelo planalto basáltico e é formado pelo acúmulo ou empilhamento de sucessivos derrames basálticos, intercalados com camadas de arenito. $\mathrm{O}$ solo da área em estudo é composto por uma camada de, aproximadamente, 2 a 3 metros de solo do tipo Latossolo Vermelho Distrófico Aluminoférrico, o qual é um solo bem drenado, normalmente variando de profundo a muito profundo (STRECK et al., 2002).

A cobertura florestal da região pertence ao domínio da Floresta Estacional Decidual e se encontra a $566 \mathrm{~m}$ de altitude (SECRETARIA ESTADUAL DO MEIO AMBIENTE, 2001). Isso porque, ao longo do Rio Uruguai e seus afluentes, estende-se uma densa floresta, caracterizada por um estrato arbóreo superior, formado por árvores altas e emergentes, na sua maioria, decidual (REITZ et al., 1988).

As quatro áreas são constituídas por: (1) floresta nativa ( $>60$ anos de idade e $15 \mathrm{ha}$ ), sobre a qual há relatos de supressão de árvores, presença de clareiras e intervenções antrópicas na área; (2) área de plantio de Eucalyptus spp. com 28 anos e 1 ha de área em espaçamento variável, com sub-bosque de vegetação nativa bem diversificada; (3) plantio agrícola (30 anos, 3 ha) com presença de Zea mays L. na área e (4) pastagem exótica com gramíneas da família Poaceae, com 10 anos de idade e 2 ha de área.

A coleta das formigas foi realizada mensalmente, durante seis meses (setembro/2008 fevereiro/2009), na área da Universidade Federal de Santa Maria campus de Frederico Westphalen - RS.

Nas áreas de floresta nativa, plantio de eucalipto e pastagem exótica, as coletas foram realizadas de setembro a janeiro. Já na área de plantio agrícola, as coletas ocorreram nos meses de setembro, outubro, novembro, janeiro e fevereiro.

Em cada coleta, foram amostrados quatro pontos aleatórios na área, com distância mínima de 20 metros da borda do sistema e 50 metros entre cada ponto. Em cada ponto, foram coletadas três amostras distantes entre si por 10 metros, sendo $12 \mathrm{~m}^{2}$ de área de serapilheira amostrada. Essas três amostras de cada ponto foram, posteriormente, misturadas, resultando em uma amostra por ponto. Assim, no final do estudo, teve-se 20 pontos coletados em cada área.

O método de extração das formigas utilizado na pesquisa é conhecido como "método Winkler de 
peneiração da serapilheira" (BESTELMEYER et al., 2000), o qual é o mais indicado para amostrar formigas de serapilheira (AGOSTI et al., 2000).

Esse método consistiu em coletar $1 \mathrm{~m}^{2}$ de serapilheira. Em seguida, a serapilheira foi peneirada por cinco minutos para desalojar a fauna. O material peneirado foi colocado em um receptáculo de malha grossa e conduzido ao laboratório para ser transferido aos extratores de mini-Winkler, que permaneceram por $72 \mathrm{~h}$ dependurados para a obtenção das formigas, no Laboratório de Proteção Florestal da Universidade Federal de Santa Maria campus de Frederico Westphalen.

As formigas foram triadas e separadas dos demais artrópodes, sendo armazenadas em frascos com álcool $70 \%$, devidamente etiquetados, para posterior identificação. Com o auxílio de lupa binocular, as formigas foram separadas por amostras e enviadas para especialistas do Laboratório de Mirmecologia do Centro de Pesquisas do Cacau/ Comissão Executiva do Plano da Lavoura Cacaueira (CEPEC/CEPLAC), Ilhéus - BA, que procederam à identificação das espécies.

Os demais artrópodes foram acondicionados no Laboratório de Proteção Florestal para posteriores avaliações.

Para a verificação de novos registros, foram consultados os catálogos de formigas neotropicais de Kempf (1972) e de Brandão (1991), e os diversos trabalhos com formigas realizados no Rio Grande do Sul.

\section{RESULTADOS E DISCUSSÃO}

Foram coletados 6300 espécimes de formigas, distribuídas em 108 espécies, 31 gêneros, 18 tribos e oito subfamílias (Amblyoponinae, Dolichoderinae, Ectatomminae, Formicinae, Heteroponerinae, Myrmicinae, Ponerinae e Proceratiinae). Destas, representantes de oito subfamílias foram encontradas na floresta nativa, cinco no plantio de eucalipto, três no plantio agrícola e três na pastagem exótica.

Das oito subfamílias coletadas, a Myrmicinae foi a que apresentou maior número de espécies $(\mathrm{S}=66)$, seguida por Formicinae ( $\mathrm{S}$ $=18)$ e Ponerinae $(\mathrm{S}=12)$. Esses resultados estão de acordo com os padrões gerais descritos para formigas, pois as três subfamílias mais ricas em espécies são consideradas as mais diversificadas dentro dos Formicidae, em todos os ambientes (HÖLLDOBLER e WILSON, 1990).
A subfamília Myrmicinae caracterizase como a maior e mais diversificada, tanto em âmbito regional quanto global, e possui adaptações ecológicas de todos os tipos (HÖLLDOBLER e WILSON, 1990), quando se trata de hábitos alimentares e nidificação (FOWLER et al., 1991). Mais de $45 \%$ das espécies e mais de $52 \%$ dos gêneros de Formicidae pertencem à subfamília Myrmicinae (BOLTON, 1995).

Do total das espécies amostradas, 90 foram coletadas na floresta nativa, seguida por plantio de eucalipto, com 65 espécies, plantio agrícola e pastagem exótica, com 20 espécies cada.

$\mathrm{Na}$ floresta nativa, coletaram-se as seguintes espécies: Amblyoponinae: Amblyopone armigera Mayr; Amblyopone elongata (Santschi); Dolichoderinae: Dorymyrmex thoracicus Gallardo; Linepithema humile (Mayr); Tapinoma sp.1; Ectatomminae: Gnamptogenys moelleri (Forel); Gnamptogenys striatula Mayr; Formicinae: Camponotus crassus Mayr; Camponotus (Myrmaphaenus) sp.1; Camponotus arboreus (Fr. Smith); Camponotus melanoticus Emery; Camponotus rufipes (Fabricius); Camponotus sericeiventris (Guérin); Brachymyrmex heeri Forel; Brachymyrmex sp.1; Brachymyrmex sp.2; Paratrechina longicornis (Latreille); Paratrechina sp.1; Paratrechina sp.2; Paratrechina (Nylanderia) sp.5; Heteroponerinae: Heteroponera dolo (Roger); Heteroponera mayri Kempf; Heteroponera sp.1; Myrmicinae: Acromyrmex laticeps Emery; Acromyrmex lundi (Guérin); Apterostigma madidiense Weber; Apterostigma sp. complexo pilosum Mayr; Apterostigma sp.1; Atta sexdens piriventris Santschi; Cyphomyrmex major Forel; Cyphomyrmex sp.1; Cyphomyrmex transversus Emery; Cyphomyrmex vorticis Weber; Myrmicocrypta sp.1; Basiceros discigera (Mayr); Basiceros sp.1; Octostruma rugifera (Mayr); Octostruma stenognatha Brown e Kempf; Wasmannia auropunctata (Roger); Wasmannia rochai Forel; Cephalotes pusillus (Klug); Crematogaster sp.1; Acanthognathus rudis (Brown e Kempf); Strumigenys (Pyramica) rugithorax (Kempf); Strumigenys cosmotela Kempf); Strumigenys silvestrii Emery; Hylomyrma balzani (Emery); Hylomyrma sp.1; Pheidole aper Forel; Pheidole heyeri Forel; Pheidole obscurithorax Naves; Pheidole radoszkoowskii Mayr; Pheidole rugatula Santschi; Pheidole siggilata Wilson; Pheidole senilis Santschi; Pheidole sospes Forel; Pheidole sp.1; Pheidole sp.2; Pheidole 
sp.3; Pheidole sp.4; Pheidole sp.5; Pheidole sp.6; Pheidole sp.7; Pheidole sp.8; Pheidole sp.9; Pheidole sp.10; Pheidole sp.16; Pheidole subarmata Mayr; Megalomyrmex drifti Kempf; Solenopsis (Diplorhoptrum) sp.1; Solenopsis richteri Forel; Solenopsis saevissima (Fr. Smith); Solenopsis sp.1; Solenopsis sp.3; Solenopsis sp.4; Solenopsis sp.5; Tetramorium bicarinatum Nylander; Ponerinae: Anochetus altisquamis Mayr; Hypoponera foreli (Mayr); Hypoponera opaciceps (Mayr); Hypoponera sp.1; Hypoponera sp.2; Hypoponera sp.3; Hypoponera sp.4; Hypoponera sp.5; Hypoponera sp.6; Pachycondyla ferruginea (Fr. Smith); Pachycondyla harpax (Fabricius); Pachycondyla striata Fr. Smith e Proceratiinae: Discothyrea sexarticulata Borgmeier.

$\mathrm{Na}$ área de plantio de eucalipto, foram coletadas as espécies: Dolichoderinae: Linepithema sp.1; Tapinoma sp.1; Formicinae: Camponotus crassus Mayr; Camponotus arboreus (Fr. Smith); Camponotus fastigatus Roger; Camponotus melanoticus Emery; Camponotus rufipes (Fabricius); Camponotus sp.1; Brachymyrmex heeri Forel; Brachymyrmex sp.1; Brachymyrmex sp.3; Paratrechina longicornis (Latreille); Paratrechina sp.1; Paratrechina sp.2; Paratrechina sp.3; Paratrechina sp.4; Myrmicinae: Acromyrmex laticeps Emery; Acromyrmex sp.1; Apterostigma madidiense Weber; Atta sexdens piriventris Santschi; Cyphomyrmex sp.2; Cyphomyrmex transversus Emery; Cyphomyrmex vorticis Weber; Trachymyrmex sp.1; Basiceros discigera (Mayr); Octostruma rugifera (Mayr); Octostruma stenognatha Brown e Kempf; Strumigenys silvestrii Emery; Hylomyrma balzani (Emery); Hylomyrma sp.1; Pheidole aper Forel; Pheidole heyeri Forel; Pheidole obscurithorax Naves; Pheidole radoszkoowskii Mayr; Pheidole rugatula Santschi; Pheidole siggilata Wilson; Pheidole sospes Forel; Pheidole sp.1; Pheidole sp.2; Pheidole sp.3; Pheidole sp.4; Pheidole sp.5; Pheidole sp.9; Pheidole sp.10; Pheidole sp.11; Pheidole sp.12; Pheidole sp.13; Pheidole sp.14; Pheidole sp.15; Pheidole subarmata Mayr; Megalomyrmex drifti Kempf; Megalomyrmex pusillus Forel; Solenopsis (Diplorhoptrum) sp.1; Solenopsis richteri Forel; Solenopsis saevissima (Fr. Smith); Tetramorium bicarinatum Nylander; Ponerinae: Hypoponera opaciceps (Mayr); Hypoponera sp.1; Hypoponera sp.2; Hypoponera sp.3; Hypoponera sp.4; Hypoponera sp.6; Pachycondyla harpax (Fabricius); Pachycondyla striata Fr. Smith e Proceratiinae:
Discothyrea sexarticulata Borgmeier.

Uma explicação para a área de plantio de eucalipto possuir alto número de espécies, segundo Braga et al. (2010), é que a área apresenta-se estruturalmente similar à floresta nativa, devido ao porte arbóreo da espécie, com presença de subbosque e serapilheira, apesar de tratar-se de um sistema monoespecífico.

A área de plantio agrícola apresentou 20 espécies: Formicinae: Camponotus crassus Mayr; Camponotus melanoticus Emery; Brachymyrmex sp.1; Paratrechina longicornis (Latreille); Myrmicinae: Crematogaster sp.1; Crematogaster sp.2; Strumigenys silvestrii Emery; Pheidole siggilata Wilson; Pheidole sp.1; Pheidole sp.2; Pheidole sp.3; Pheidole sp.4; Pheidole subarmata Mayr; Solenopsis saevissima (Fr. Smith); Solenopsis sp.1; Solenopsis sp.2; Tetramorium bicarinatum Nylander; Hypoponera opaciceps (Mayr); Hypoponera sp.1; Pachycondyla striata Fr. Smith.

A área de plantio agrícola com 20 espécies de formigas coletadas evidencia que a diversidade de insetos está positivamente associada à diversidade de plantas perenes e negativamente associada à diversidade de plantas anuais (DEAN e MILTON, 1995).

$\mathrm{Na}$ área de pastagem exótica, foram coletadas: Paratrechina longicornis (Latreille); Paratrechina sp.1; Paratrechina sp.2; Atta sexdens piriventris Santschi; Wasmannia auropunctata (Roger); Crematogaster sp.1; Strumigenys silvestrii Emery; Pheidole rugatula Santschi; Pheidole sp.2; Pheidole sp.3; Pheidole sp.4; Pheidole sp.17 grupo fallax; Solenopsis (Diplorhoptrum) sp.1; Solenopsis richteri Forel; Solenopsis saevissima (Fr. Smith); Tetramorium bicarinatum Nylander; Hypoponera sp.1; Hypoponera sp.2; Hypoponera sp.3; Hypoponera sp.4.

Segundo Andersen (2000), a riqueza de espécies de formigas aumenta com o aumento da complexidade estrutural do habitat. Em ambientes tropicais, a riqueza de espécies é maior em florestas e menor em formações vegetais mais homogêneas, como áreas cultivadas e campos de gramíneas (LEAL e LOPES, 1992).

O plantio de eucalipto, muitas vezes implementado em substituição às pastagens degradadas, apesar de constituir uma monocultura, pode resultar em ganho para a biodiversidade, devido a sua complexidade estrutural comparada à pastagem (PERFECTO e SNELLING, 1995). Porém, o valor dos agroecossistemas florestais 
para a conservação de espécies ainda se encontra negligenciado (SPERBER et al., 2004).

Fonseca e Diehl (2004), estudando a riqueza de formigas em povoamentos de Eucalyptus spp. de diferentes idades em Capivari do Sul, encontraram reduzida riqueza de espécies e a associaram à baixa complexidade estrutural da vegetação local, expressa pela ausência de sub-bosque. Essa condição é contrária à da área do presente estudo, que possui sub-bosque diversificado.

As espécies comuns aos quatro ambientes foram: Paratrechina longicornis Latreille, Strumigenys silvestrii Emery, Pheidole sp.2, Pheidole sp.3, Pheidole sp.4, Solenopsis saevissima Smith, Tetramorium bicarinatum Nylander e Hypoponera sp.1.

$\mathrm{O}$ gênero Pheidole apresentou maior riqueza específica dentro da subfamília Myrmicinae, com 26 espécies coletadas no total, seguida por Camponotus e Hypoponera, com oito espécies cada. Situação semelhante foi encontrada em fragmentos florestais, em Santa Maria - RS, por Ribeiro (2011), que coletou 14 espécies do gênero Camponotus, 10 espécies de Hypoponera e nove espécies de Pheidole.

Esses gêneros, de acordo com Wilson (1976), estão entre os mais representativos em escala mundial, devido a características como distribuição geográfica extensa, dieta flexível, adaptações diversas, grande capacidade colonizadora e abundância local, além de apresentarem os maiores números de espécies.

Solenopsis saevissima (Fr. Smith), registrada como a espécie mais frequente no geral (1343 registros) e também na área de floresta nativa (876 registros) e na área de plantio de eucalipto (419 registros), pertence a um gênero considerado dominante na serapilheira (DELABIE; AGOSTI; NASCIMENTO, 2000). As espécies desse gênero são encontradas nos mais diversos habitats, desde florestais preservados (DELABIE e FOWLER, 1995), áreas de recuperação florestal (RAMOS et al., 2003) até ambientes mais abertos, como agrícolas, por possuírem alta capacidade de invasão e adaptação às áreas com impacto antrópico (DELABIE e FOWLER, 1995; RAMOS et al., 2003; PACHECO et al., 2009). Possuem, ainda, dieta alimentar variada, podendo passar longos períodos de escassez de alimento, assim como eficiente recrutamento em massa, competindo com outras espécies de formigas e outros grupos de animais por recursos (FOWLER et al., 1991).
Um total de 37 espécies das 108 coletadas ocorreu somente na área de floresta nativa, perfazendo a maior fauna exclusiva dentre as quatro áreas estudadas. Uma das espécies exclusivas, Anochetus altisquamis Mayr, é críptica, típica de serapilheira e característica de ambientes florestais (SILVESTRE e SILVA, 2001; FERNÁNDEZ, 2003; LATTKE, 2003), sendo considerada importante para esse ecossistema (MAJER e NICHOLS, 1998) e típica de interior de floresta, com maior cobertura vegetal (HÖLLDOBLER e WILSON, 1990).

Dentre as 108 espécies coletadas no presente estudo, 20 espécies não estavam citadas nos catálogos de Kempf (1972) e de Brandão (1991), como de ocorrência para o Rio Grande do Sul, nem em trabalhos realizados no estado (HAMEISTER; DIEHL-FLEIG; DIEHL， 2003; FONSECA; DIEHL, 2004; SACCHET; DIEHL, 2004; DIEHL; SANHUDO; DIEHL-FLEIG, 2004; DIEHL; SACCHET; ALBUQUERQUE, 2005; MARCHIORETTO; DIEHL, 2006; SCHMIDT; DIEHL, 2008; ALBUQUERQUE e DIEHL, 2009; BOSCARDIN et al., 2011; FRÖHLICH et al., 2011; RIBEIRO, 2011; ROSADO et al., 2012; BOLICO et al., 2012). Dessa forma, este é o primeiro registro dessas espécies para o Rio Grande do Sul (Tabela 1). Salienta-se que algumas dessas espécies, consideradas como registros novos para o estado, podem ser morfoespécies já registradas em outros trabalhos. Isso corrobora a informação de Brandão (1999), que enfatiza não haver uma listagem confiável de espécies, pois, para várias regiões do Brasil, não foram executados levantamentos faunísticos.

Em Santa Catarina, foi realizada uma listagem bem atual e pormenorizada das formigas registradas, sendo este o único estado brasileiro que possui uma listagem desse tipo (ULYSSÉA et al., 2011).

As 20 espécies de formigas consideradas como primeiro registro para o estado do Rio Grande do Sul estão distribuídas em quatro subfamílias (Dolichoderinae, Formicinae, Myrmicinae e Ponerinae), nove tribos e 10 gêneros. Destas, na floresta nativa, registraram-se 19 espécies, sendo nove delas espécies exclusivas, sendo que a única espécie não coletada foi Megalomyrmex pusillus Forel, a qual é exclusiva do plantio de eucalipto. Já na área de plantio de eucalipto, foram encontradas 11 espécies que são consideradas de primeiro registro.

Uma das espécies exclusivas da floresta nativa foi Wasmannia rochai Forel. O gênero 


\begin{tabular}{|c|c|c|c|c|}
\hline \multirow{2}{*}{ Espécies de Formicidae } & \multicolumn{4}{|c|}{ Espécimes } \\
\hline & Floresta nativa & Plantio de eucalipto & Plantio agrícola & Pastagem exótica \\
\hline \multicolumn{5}{|l|}{ Subfamília Dolichoderinae } \\
\hline \multicolumn{5}{|l|}{ Tribo Dolichoderini } \\
\hline Dorymyrmex thoracicus Gallardo & 4 & 0 & 0 & 0 \\
\hline \multicolumn{5}{|l|}{ Subfamília Formicinae } \\
\hline \multicolumn{5}{|l|}{ Tribo Camponotini } \\
\hline Camponotus arboreus (Fr. Smith) & 3 & 18 & 0 & 0 \\
\hline Camponotus melanoticus Emery & 9 & 39 & 3 & 0 \\
\hline \multicolumn{5}{|l|}{ Subfamília Myrmicinae } \\
\hline \multicolumn{5}{|l|}{ Tribo Attini } \\
\hline Apterostigma madidiense Weber & 58 & 2 & 0 & 0 \\
\hline Apterostigma sp. complexo pilosum Mayr & 1 & 0 & 0 & 0 \\
\hline Cyphomyrmex major Forel & 2 & 0 & 0 & 0 \\
\hline Cyphomyrmex vorticis Weber & 3 & 28 & 0 & 0 \\
\hline \multicolumn{5}{|l|}{ Tribo Blepharidattini } \\
\hline Wasmannia rochai Forel & 2 & 0 & 0 & 0 \\
\hline \multicolumn{5}{|l|}{ Tribo Cephalotini } \\
\hline Cephalotes pusillus (Klug) & 1 & 0 & 0 & 0 \\
\hline \multicolumn{5}{|l|}{ Tribo Dacetini } \\
\hline Strumigenys (Pyramica) rugithorax (Kempf) & 1 & 0 & 0 & 0 \\
\hline Strumigenys cosmotela Kempf & 1 & 0 & 0 & 0 \\
\hline Strumigenys silvestrii Emery & 39 & 105 & 9 & 2 \\
\hline \multicolumn{5}{|l|}{ Tribo Pheidolini } \\
\hline Pheidole aper Forel & 10 & 3 & 0 & 0 \\
\hline Pheidole heyeri Forel & 5 & 19 & 0 & 0 \\
\hline Pheidole rugatula Santschi & 54 & 92 & 0 & 6 \\
\hline Pheidole siggilata Wilson & 38 & 34 & 29 & 0 \\
\hline Pheidole senilis Santschi & 1 & 0 & 0 & 0 \\
\hline Pheidole sospes Forel & 58 & 6 & 0 & 0 \\
\hline \multicolumn{5}{|l|}{ Tribo Solenopsidini } \\
\hline Megalomyrmex pusillus Forel & 0 & 5 & 0 & 0 \\
\hline \multicolumn{5}{|l|}{ Subfamília Ponerinae } \\
\hline \multicolumn{5}{|l|}{ Tribo Ponerini } \\
\hline Pachycondyla ferruginea (Fr. Smith) & 1 & 0 & 0 & 0 \\
\hline Total de gêneros & 9 & 6 & 3 & 2 \\
\hline Total de espécies & 19 & 11 & 3 & 2 \\
\hline Total de espécimes & 291 & 351 & 41 & 8 \\
\hline
\end{tabular}

Wasmannia é um conhecido indicador de áreas degradadas e, geralmente, domina o meio onde se instala, influenciando na composição da mirmecofauna local (ALVES, 2007). Isso pode ter ocorrido devido à área de floresta nativa em estudo estar sofrendo ação antrópica, à semelhança do que ocorreu na Praia da Pedreira, no Parque Estadual de Viamão, que registrou essa espécie na mata nativa. Apesar de a área sofrer ação antrópica, ela ainda conserva uma mirmecofauna bastante rica (DIEHL; SACCHET e ALBUQUERQUE, 2005).

Outros estudos já encontraram espécies que correspondem a registros novos para o Rio Grande do Sul, como os de Gonçalves (1961), que registraram a subespécie Acromyrmex rugosus rugosus; os de Diehl, Sacchet e Albuquerque (2005), na Praia da Pedreira; os de Diehl et al. (2005), na Floresta Nacional de São Francisco de 
Paula; os de Diehl e Albuquerque (2007), nas quatro províncias geomorfológicas do Rio Grande do Sul; os de Albuquerque e Diehl (2009), em campo nativo no Planalto das Araucárias, e os de Rosado et al. (2012), em vinhedos e áreas de pastagens na região da Campanha.

\section{CONCLUSÕES}

Relatou-se o primeiro registro de ocorrência, para o estado do Rio Grande do Sul, das seguintes espécies de formigas: Dorymyrmex thoracicus (Dolichoderinae), Camponotus arboreus, Camponotus melanoticus (Formicinae), Apterostigma madidiense, Apterostigma sp. complexo pilosum, Cyphomyrmex major, Cyphomyrmex vorticis, Wasmannia rochai, Cephalotes pusillus, Strumigenys (Pyramica) rugithorax, Strumigenys cosmotela, Strumigenys silvestrii, Pheidole aper, Pheidole heyeri, Pheidole obscurithorax, Pheidole rugatula, Pheidole siggilata, Pheidolesenilis, Pheidole sospes, Pheidole subarmata, Megalomyrmex pusillus (Myrmicinae) e Pachycondyla ferruginea (Ponerinae).

\section{AGRADECIMENTOS}

Ao pesquisador Jacques Hubert Charles Delabie do Laboratório de Mirmecologia do Centro de Pesquisas do Cacau/Comissão Executiva do Plano da Lavoura Cacaueira (CEPEC/CEPLAC), Ilhéus - BA, pela identificação das espécies de formigas.

\section{REFERÊNCIAS BIBLIOGRÁFICAS}

AGOSTI, D. et al. Ants: standard methods for measuring and monitoring biodiversity. Washington: Smithsonian Institution Press, 2000.

ALBUQUERQUE, E. Z.; DIEHL, E. Análise faunística das formigas epígeas (Hymenoptera: Formicidae) em campo nativo no planalto das Araucárias, Rio Grande do Sul. Revista Brasileira de Entomologia, Curitiba, v. 53, n. 3, p. 398-403, 2009.

ALVES, H. S. R. Identificação de bioindicadores e planejamento de mini-corredores ecológicos na área de proteção ambiental Costa de Itacaré/ Serra Grande, Bahia, Ilhéus, BA. 2007. Dissertação (Mestrado em Desenvolvimento Regional e Meio Ambiente) - Universidade Estadual de Santa Cruz, Bahia, 2007.
ANDERSEN, A. N. A global ecology of rainforest ants: functional groups is relation to environmental stress and disturbance. In: AGOSTI, D. et al. (Org.). Ants: standard methods for measuring and monitoring biodiversity. Washington: Smithsonian Institution Press, 2000. p. 25-34.

BESTELMEYER, B. T. et al. Field techniques for the study of ground-dwelling ants - an overview, description, and evaluation. In: AGOSTI, D. et al. (Org.). Ants: standard methods for measuring and monitoring biodiversity. Washington: Smithsonian Institution Press, 2000. p. 122-144.

BOLICO, C. F. et al. Mirmecofauna (Hymenoptera, Formicidae) de duas marismas do Estuário da Lagoa dos Patos, RS: diversidade, flutuação de abundância e similaridade como indicadores de conservação. EntomoBrasilis, Vassouras, v. 5, n. 1, p. 11-20, 2012. Disponível em: <http://www.periodico. ebras.bio.br/ojs/index.php/ebras/article/view/147>. Acesso em: 11 fev. 2013.

BOLTON, B. A taxonomic and zoogeographical census of the extant ant taxa. Journal of Natural History, v. 29, p. 1037-1056, 1995.

BOSCARDIN, J. et al. Avaliação comparativa de iscas atrativas a partir da riqueza de espécies de formigas (Hymenoptera: Formicidae) numa floresta de Eucalyptus grandis, em Santa Maria, Rio Grande do Sul, Brasil. AUGMDOMUS, La Plata, v. 3, p. 10-19, 2011. Disponível em: $<$ http://revistas.unlp. edu.ar/index.php/domus/issue/current/showtoc $>$. Acesso em: 11 fev. 2013.

BRAGA, D. L. et al. Avaliação rápida da diversidade de formigas em sistemas de uso do solo no sul da Bahia, Neotropical Entomology, Londrina, v. 39, n. 4, p. 464-469, 2010.

BRANDÃO, C. R. F. Adendos ao catálogo abreviado das formigas da região neotropical (Hymenoptera: Formicidae). Revista Brasileira de Entomologia, Curitiba, v. 35, n. 2, p. 319-412, 1991.

BRANDÃO, C. R. F. Família Formicidae. São Paulo, 1999. Disponível em $<$ http:// www.biota.org. br/pdf/v5cap21.pdf $>$. Acesso em: 22 mar. 2012.

DEAN, W. R. J.; MILTON, S. J. Plant and invertebrate assemblages on old fields in the arid southern Karoo, South Africa. African Journal of Ecology, v. 33, p. 1-13, 1995.

DELABIE, J. H. C.; FOWLER, H. G. Soil and litter cryptic ant assemblages of bahian cocoa plantations. Pedobiologia, v. 39, p. 423-433, 1995.

DELABIE, J. H. C.; AGOSTI, D.; NASCIMENTO, I. C. Litter and communities of the Brazilian Atlantic rain forest region. In: AGOSTI, D. et al. Sampling 
ground - dwelling ants: case studies from the world's rain forest. Perth: Curtin University School of Environmental Biology, 2000. p. 1-17.

DIEHL, E.; ALBUQUERQUE, E. Z. Representantes das quatro províncias geomorfológicas do Rio Grande do Sul na coleção de Formicidae do Laboratório de Insetos Sociais da Unisinos. Biológico, São Paulo, v. 69, n. 2, p. 101-104, 2007. DIEHL, E. et al. Riqueza e composição das comunidades de formigas e de térmitas na Floresta Nacional de São Francisco de Paula (FLONA-SFP), RS. Acta Biologica Leopondensia, São Leopoldo, v. 27, n. 2, p. 99-106, 2005.

DIEHL, E.; SACCHET, F.; ALBUQUERQUE, E. Z. de. Riqueza de formigas de solo, na Praia da Pedreira, Parque Estadual de Itapuã, Viamão, RS, Brasil. Revista Brasileira de Entomologia, Curitiba, v. 49, n. 4, p. 552-556, dez. 2005.

DIEHL, E.; SANHUDO, C. E. D.; DIEHL-FLEIG, E. Ground-dwelling ant fauna of sites with high levels of copper. Brazilian Journal of Biology, São Carlos, v. 64, n. 1, p. 33-39, 2004.

FERNÁNDEZ, F. Subfamilia Myrmicinae. In: FERNÁNDEZ, F. (Org.). Introducción a las hormigas de la región Neotropical. Bogotá, Colômbia: Instituto de Investigación de Recursos Biológicos Alexander Von Humboldt, 2003. Cap. 22, p. 307-330.

FITTKAU, E. J.; KLINGE, H. On biomass and trophic structure of the Central Amazonian rain forest ecosystem. Biotropica, Oxford, v. 5, n. 1, p. 2-14, 1973.

FONSECA, R. C.; DIEHL, E. Riqueza de formigas (Hymenoptera, Formicidae) epigéicas em povoamentos de Euclyptus spp. (Myrtacea) de diferentes idades no Rio Grande do Sul, Brasil. Revista Brasileira de Entomologia, Curitiba, v. 48, n. 1, p. 95-100, mar. 2004.

FOWLER, H. G. et al. Ecologia nutricional de formigas. In: PANIZZI, A. R.; PARRA, J. R. P. (Org.). Ecologia nutricional de insetos e suas implicações no manejo de pragas. São Paulo: Manole, 1991. p. 131-223.

FRÖHLICH, F. R. da S. et al. Diversidade de formigas (Formicidae) em áreas de eucalipto e vegetação nativa no município de Capitão, Rio Grande do Sul. Caderno Pedagógico, Lajeado, v. 8, n. 2, p. 109-124, 2011.

GONÇALVES, C. R. O gênero Acromyrmex no Brasil (Hymenoptera: Formicidae). Studia Entomologica, Petrópolis, v. 4, n. 1, p. 113-180, 1961.
HAMEISTER, T. M.; DIEHL, FLEIG, E.; DIEHL, E. Comunidades de formigas (Hymenoptera: Formicidae) epígeas no morro de Itapeva, município de Torres, RS. Acta Biologica Leopoldensia, São Leopoldo, v. 25, n. 2, p. 187-195, jul/dez. 2003.

HÖLldoBler, B.; WILSON, E. O. The Ants. Cambridge: Harvard University Press, 1990. 732 p. KEMPF, W. W. Catálogo abreviado das formigas da região Neotropical (Hymenoptera: Formicidae). Studia Entomologica, Petrópolis, v. 15. n. 1-4, p. 3-344, 1972.

LATTKE, J. E. Subfamilia Ponerinae. In: FERNÁNDEZ, F. (Org.). Introducción a las hormigas de la región Neotropical. Bogotá, Colômbia: Instituto de Investigación de Recursos Biológicos Alexander Von Humboldt, 2003. Cap. 16, p. 261-276.

LEAL,I.R.;LOPES,B.C. Estrutura das comunidades de formigas (Hymenoptera: Formicidae) de solo e vegetação no Morro da Lagoa da Conceição, Ilha de Santa Catarina, SC. Biotemas, Florianópolis, v. 5, p. 107-122, 1992.

MAJER, J. D.; NICHOLS, O. G. Long-term recolonization patterns of ants in Western Australia rehabilitated bauxite mines with reference to their use as indicators of restoration success. Journal of Applied Ecology, London, v. 35, p. 161-182, 1998. MARCHIORETTO, A.; DIEHL, E. Distribuição espaço temporal de uma comunidade de formigas em um remanescente de floresta inundável às margens de um meandro antigo do Rio dos Sinos, São Leopoldo, RS. Acta Biologica Leopoldensia, São Leopoldo, v. 28, n. 1, p. 25-31, 2006.

MORENO, J. A. Clima do Rio Grande do Sul. Porto Alegre: Secretaria da Agricultura, 1961. 83 p. PACHECO, R. et al. A comparison of the leaf litter ant fauna in a secondary Atlantic Forest with an adjacent pine plantation in southeastern Brazil. Neotropical Entomology, Londrina, v. 38, n. 1, p. 55-65, 2009.

PERFECTO, I.; SNELLING, R. Biodiversity and the transformation of tropical agroecosystem: ants in coffee plantations. Ecological Applications, v. 5, p. 1084-1097, 1995.

RAMOS, L. S. et al. Comunidades de formigas (Hymenoptera: Formicidae) de serapilheira em áreas de cerrado "stricto sensu" em Minas Gerais. Lundiana, Belo Horizonte, v. 4, n. 2, p. 95-102, 2003.

REITZ, R. et al. Projeto Madeira do Rio Grande do Sul. Porto Alegre: SUDESUL-GERS-IBDF, 1988. 
RIBEIRO, T. C. Diversidade de formigas (Hymenoptera: Formicidae) de fragmentos florestais em Santa Maria, Rio Grande do Sul, Brasil. 2011. Dissertação (Mestrado em Ciências Biológicas) - Universidade Federal de Santa Maria, Santa Maria, 2011.

ROSADO, J. L. O. et al. Epigeic ants (Hymenoptera: Formicidae) in vineyards and grassland areas in the Campanha region, state of Rio Grande do Sul, Brazil. Check List, v. 8, n. 6, p. 1184-1189, 2012.

SACCHET, F.; DIEHL, E. Comunidades de formigas de solo no Morro da Grota, Parque Estadual de Itapuã, RS. Acta Biologica Leopoldensia, São Leopoldo, v. 26, n. 1, p. 79-92, jan/jun. 2004

SCHMIDT, F. A.; DIEHL, E. What is the Effect of Soil Use on Ant Communities? Neotropical Entomology, Londrina, v. 37, n. 4, p. 381-388, 2008.

SECRETARIA ESTADUAL DO MEIO AMBIENTE. Relatório Final do Inventário Florestal Contínuo do Rio Grande do Sul. Porto Alegre, 2001. 706 p.
SILVESTRE, R.; SILVA, R. R. Guildas de formigas da Estação Ecológica Jataí, Luís Antonio - SP - sugestões para aplicação de guildas como bioindicadores ambientais. Biotemas, Florianópolis v. 14, n. 1, p. 37-69, 2001.

SPERBER, C. F. et al. Tree species richness and density affect parasitoid diversity in cacao agroforestry. Basic Applied Ecology, v. 5, p. 241-251, 2004.

STRECK, E. V. et al. Solos do Rio Grande do Sul. Porto Alegre: EMATER/RS, 2002. 107 p.

ULYSSÉA, M. A. et al. Updated list of ant species (Hymenoptera, Formicidae) recorded in Santa Catarina State, Southern Brazil, with a discussion of research advances and priorities. Revista Brasileira de Entomologia, Curitiba, v. 55, n. 4, p. 603-611, 2011.

WILSON, E. O. Wich are the most prevalent ant genera? Studia Entomologica, v. 19, p. 187-200, 1976.

WILSON, E. O. Causes of ecological success: the case of the ants. Journal of Animal Ecology, London, v. 56, p. 1-9, 1987. 